\title{
An examination of the effects of mammillary-body lesions on reversal learning sets in monkeys
}

\author{
EDWARD J. HOLMES and NELSON BUTTERS \\ Boston VA Medical Center and Boston University School of Medicine, Boston, Massachusetts \\ and \\ STANLEY JACOBSON and BENNETT M. STEIN
Tufts University and Tufts New England Medical Center, Boston, Massachusetts
}

\begin{abstract}
Monkeys with mammillary-body lesions were compared with operated and unoperated control monkeys on the acquisition of a series of place and object reversal discriminations. The animals with mammillary-body lesions were found not to differ from the control animals on either the initial acquisition of a place response or the visual discrimination of two junk objects. On subsequent reversals, however, monkeys with mammillary-body lesions were severely impaired on the spatial task, but showed no impairment in reversal learning in the object task. These results are consistent with our previous findings of an impairment in spatial alternation learning following mammillary-body lesions in monkeys and supports the hypothesis that the role of the mammillary nuclei in cognition may be limited to the spatial domain.
\end{abstract}

In man, damage to the mammillary nuclei of the hypothalamus has been associated with an array of memory and other cognitive impairments. Patients with alcoholic Korsakoff's syndrome, whose neuropathology usually involves both the mammillary nuclei and the dorsomedial nuclei of the thalamus, have severe anterograde and retrograde amnesias (Butters \& Cermak, 1980; Victor, Adams, \& Collins, 1971), visuoperceptive difficulties (Kapur \& Butters, 1977), and deficits in shifting cognitive strategies on problemsolving tasks (Oscar-Berman, Sahakian, \& Wikmark, 1976; Oscar-Berman \& Zola-Morgan, 1980). Despite the regularity with which the mammillary nuclei have been linked to such debilitating cognitive changes in man, there is little experimental evidence demonstrating this association between structure and function in animals. Thompson and his colleagues (1964) have reported that bilateral lesions of the mammillary nuclei in rats produces an impairment in learning to reverse

This research was supported in part by funds from the Medical Research Service of the Veterans Administration and in part by NIAAA Grant AA-00187 to Boston University School of Medicine. During the course of the research, E. J. Holmes and N. Butters were associated with the Psychology Service of the Boston VA Medical Center and the Neurology Department of Boston University School of Medicine; S. Jacobson and B. M. Stein were associated with the Departments of Anatomy and Neurosurgery at their two institutions. Requests for reprints should be sent to: Dr. Edward J. Holmes, Rose Kennedy Center for Mental Retardation, Rm. 322, Department of Neuroscience, Albert Einstein College of Medicine, 1300 Morris Park Ave., Bronx, New York 10461 . acquired spatial responses in a T-maze, although the memory for such spatial responses was reported to remain normal (Thompson, 1981). Similarly, in a recent study with monkeys, Holmes, Jacobson, Stein, and Butters (1983) found a marked impairment on the acquisition of a spatial delayed alternation task following mammillary-body damage, although there was no impairment in the animals' ability to retain the solution to three visuospatial tasks acquired before surgery. This limitation of learning deficits to the spatial domain in both rats and monkeys tends to support the contention that damage to the mammillary nuclei alone is insufficient to produce the severe and general cognitive changes that are associated with alcoholic Korsakoff's syndrome.

To further examine the generality of the learning deficit associated with lesions limited to the mammillary nuclei, monkeys with bilateral mammillarybody lesions were compared with operated and unoperated control monkeys on the postoperative acquisition of a series of place and object reversal discriminations. A spatial reversal deficit combined with normal performance on the object reversal task would provide further evidence that the function of the mammillary nuclei in memory may be restricted to the spatial domain.

\section{METHOD}

\section{Subjects}

The subjects were 10 cynomolgus (M. fasicularis) monkeys. Using a subtemporal approach to the base of the hypothalamus, 
4 monkeys received unilateral third cranial nerve transections as well as bilateral lesions of the mammillary nuclear complex. Three monkeys served as operated control subjects and received unilateral transections of the third cranial nerve alone. The remaining three monkeys remained unoperated. All of the subjects were equal in their previous training experience.

\section{Surgery}

Before surgery, the subjects were initially anesthetized with an intramuscular injection $(30 \mathrm{mg} / \mathrm{kg})$ of ketamine hydrochloride. The animal's head was shaven and secured in a primate headholder and tilted approximately $60 \mathrm{deg}$ to one side. Surgery was conducted under asceptic conditions with the subject further anesthetized with intravenous injections of sodium pentobarbitol (approximately 0.3-0.4 cc), as needed. Mannitol was also administered $(1.5 \mathrm{~g} / \mathrm{kg}$, intravenously) to reduce brain tissue fluids during surgery.

A detailed account of the microsurgical procedures has been presented elsewhere (Holmes et al., 1983). Briefly, the surgical approach to the mammillary nuclei consisted of entering beneath the temporal lobe following the gentle elevation of the temporal pole. Under microscopic enhancement, the third cranial nerve and the internal carotid artery were used as guides to the base of the hypothalamus. The ipsilateral third cranial nerve was subsequently transected to allow additional temporal lobe retraction to reveal the arachnoidal cisterns and the median eminence. The mammillary nuclei were estimated to be localized just posterior to the median eminence and the origin of the pituitary stalk. The nuclei were destroyed bilaterally via a radiofrequency current which was delivered through a thin wire electrode (completely insulated except at the tip). Under direct visual examination, enough current (approximately $60 \mathrm{~mA}$ ) was delivered through the electrode to cauterize the target area. Several electrode penetrations were made within the nuclear complex of each hemisphere until the-lateral, medial, and intermediate nuclei of each mammillary body were believed to be completely destroyed. Following the completion of the lesions, the dura mater, muscle, fascia, and skin were sutured in anatomical layers. The subject was then given 600,000 units of penicillin and placed in a warm area to recover. Recovery from surgery was uneventful in each case.

The unilateral third cranial nerve transections were later noted to result in ptosis, loss of pupillary accommodation, and aberrant movements of the ipsilateral eye. Although the ptosis completely disappeared in most of the cases, the pupillary dilation and aberrant eye movements were permanent. We cannot comment at this time on the functional capacity of the damaged eye.

\section{Behavioral Apparatus}

All behavioral training was conducted in a Wisconsin General Test Apparatus (WGTA), in which an opaque screen separated an illuminated compartment containing the animal's cage from a second illuminated compartment containing a gray test board. In the test board were two foodwells that were $35.5 \mathrm{~cm}$ apart (center to center) and located $15.5 \mathrm{~cm}$ from the animal's cage. A one-way screen allowed the experimenter to observe the subject's response when the opaque screen was raised. Training was conducted in a darkened, ventilated room with masking noise present.

\section{Training Procedures}

Spatial reversal learning. In the spatial reversal task, both foodwells were covered by identical yellow Plexiglas plaques. The monkeys were initially trained (while partially food deprived) for $\mathbf{5 0}$ trials per day, 7 days each week, to displace the plaque covering the left foodwell in order to obtain a food reward (lightly sweetened cereal). Once the animals reached a criterion level of 27 correct responses in 30 consecutive trials, the reward was switched to the right foodwell and the subjects were required to reverse their responses accordingly. The monkeys were trained to respond to the right foodwell until they again reached a criterion level of 27 correct responses in 30 consecutive trials. Subsequent reversals were continued until the animals were able to successfully reverse a learned spatial response within one training session and with no more than 3 errors in the first 30 reversal trials. Training was continued until the monkeys reached the learning set criterion or for a maximum of 20 reversals.

Object reversal learning. The procedures for the object reversal task were similar to those followed in the spatial reversal task except that the foodwells were covered by two different junk objects which were similar in size, but differed markedly in form and color. The monkeys were trained for 50 trials per day to displace one of the objects for a food reward regardless of the object's left-right spatial position on the test board. The animals were trained on this task until a learning criterion of 27 correct responses in 30 consecutive trials was attained. After reaching criterion performance on the first task, the animals were then trained to reverse their responses to the alternate object until they again reached a learning criterion of 27 correct responses in 30 consecutive trials. Subsequent reversals were continued until the monkeys were able to successfully reverse responses within one training session with no more than 3 errors in the first $\mathbf{3 0}$ reversal trials. Training was continued until the monkeys reached the learning set criterion or for a maximum of 20 reversals.

\section{Histological Procedures}

At the conclusion of behavioral training, the experimental and operated control animals were deeply anesthetized with an intraperitoneal injection of sodium pentobarbitol and perfused through the heart with $9 \%$ saline followed by $10 \%$ Formalin. The brains were subsequently extracted and placed in a $30 \%$ sucrose-Formalin solution for approximately 7 to 10 days. After the brain tissue finally sank to the bottom of the solution, frozen sections within and surrounding the hypothalamic region were taken in the coronal plane at a thickness of $40 \mu \mathrm{m}$. The sections were later mounted onto glass slides and stained with thionin to differentiate cell bodies from fiber tracts.

\section{RESULTS}

\section{Histology}

Histological examinations of the brains of the experimental subjects revealed that the lesions of the mammillary bodies were accurate and complete in four out of four animals. The extent of the damage to the mammillary nuclei varied from shrunken cellular areas and the disruption of neuronal organization to the complete loss of cellular tissue. The extent of the hypothalamic lesion in two subjects is given in Figure 1. In each experimental case, no additional or inadvertent damage from surgery was noted in neighboring areas of the diencephalon or in the brainstem and no anterograde degeneration was observed in the anterior thalamic nuclei. In both the experimental and operated control groups, however, it was noted that the retraction of the temporal pole in the surgical approach to the base of the brain invariably resulted in minor unilateral damage (infarct) in the anterior temporal area, which frequently encroached dorsally upon the lateral and basal (pars lateralis) nuclei of the amygdaloid complex (divisions according to Snider \& Lee, 1961). Ventrally, damage was noted in some of the fibers and adjoining inner cortical layers in the ventral rhinencephalic area. Nevertheless, in none of the cases was any damage inflicted to the anterior hippocampus or the inferior temporal 

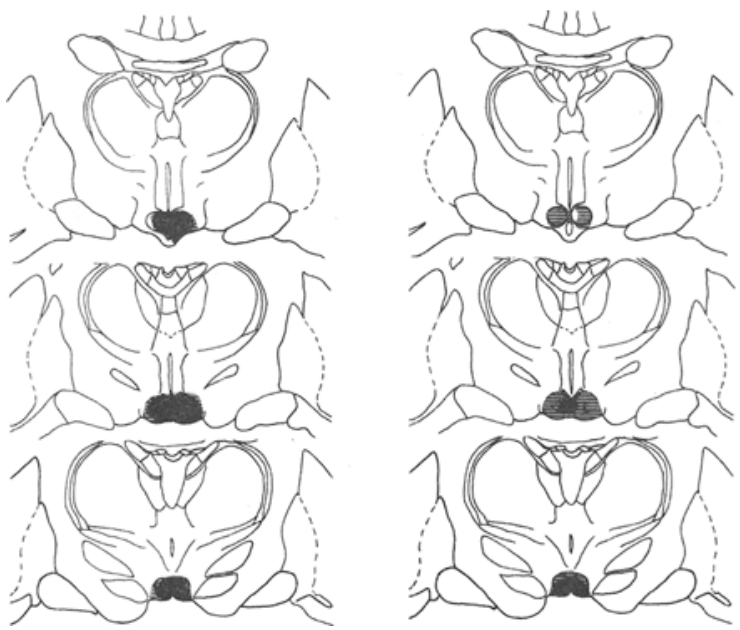

Figure 1. Histological reconstructions of the largest (left) and smallest (right) lesion of the mammillary nuclei in two animals. Three levels (anterior to posterior) through the mammillary nuclei are shown for each case. Blackened areas represent either the loss of tissue or areas totally devoid of cells. Cross-hatched areas represent tissue characterized by shrunken cells, gliosis, and disrupted cellular organization.

neocortex, both of which are believed to be involved in learning and memory processes (Gross, 1973; O'Keefe \& Nadel, 1978).

\section{Behavior}

The results of the spatial reversal task are shown in Figure 2. As indicated in the figure and outlined for each animal in Table 1, the monkeys with mammillary-body lesions did not differ significantly from either the operated $(U=3, p>.10$, Mann-Whitney $U$ test $)$ or the unoperated $(U=5, p>.10$, MannWhitney $U$ test) control subjects in the initial acquisition of a position response habit to the left side of the test board. On the subsequent reversals, however, as is evident in Figure 2, the monkeys with mammillarybody lesions were significantly impaired in acquiring the set reversal task relative to the performance of the two control groups, which, in turn, did not differ from one another. Using a repeated-measures design for unweighted means, an analysis of variance for the reversal data (trials to criterion) revealed a significant lesion effect $[F(2,7)=23.63, p<.01]$, a significant effect across reversals $[F(7,49)=53.16$, $\mathrm{p}<.01$ ], and a significant lesion $\times$ reversal interaction $[F(14,49)=5.52, p<.01]$. It should be noted that because of the unequal number of reversals required by each group to attain the preset learning criterion, the analysis of variance was based only on the first eight reversals. In addition, although the animals with mammillary-body lesions were impaired in the acquisition of this task, all but one of the subjects was able to attain the preset learning criterion within 20 reversals.

The data for the individual animals on the spatial reversal task are provided in Table 1 in blocks of four reversals. As shown in the table, although the animals in the two control groups consistently overlap in performance, no overlap was obtained between the control subjects and the monkeys with mammil-

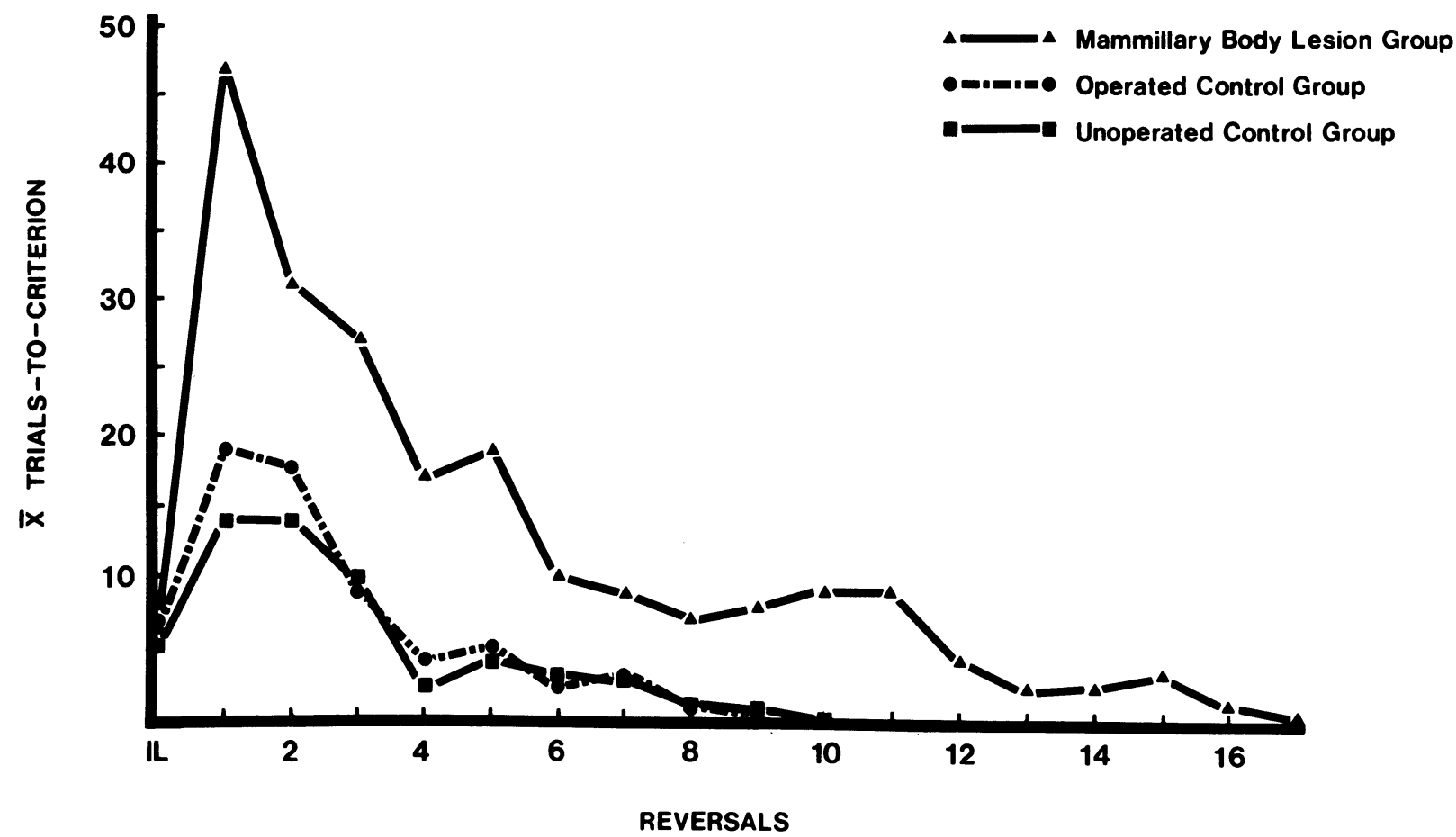

Figure 2. Spatial reversal learning curves. Abbreviations: $\mathbf{I L}=$ initial learning. 
Table 1

Spatial Reversal Learning

\begin{tabular}{|c|c|c|c|c|c|c|c|}
\hline \multirow[b]{2}{*}{ Subject } & \multirow{2}{*}{$\begin{array}{c}\text { Initial Learning } \\
\text { Trials to Criterion }\end{array}$} & \multicolumn{5}{|c|}{$\begin{array}{l}\text { Blocks of Four Reversals } \\
\text { Trials to Criterion }\end{array}$} & \multirow{2}{*}{$\begin{array}{c}\text { Total Reversals to } \\
\text { Learning Set Criterion }\end{array}$} \\
\hline & & 1 & 2 & 3 & 4 & 5 & \\
\hline \multicolumn{8}{|c|}{ Mammillary Body Lesion Group } \\
\hline 1 & 3 & 35.0 & 15.3 & 8.3 & 2.0 & & 15 \\
\hline 2 & 2 & 34.5 & 12.8 & 6.0 & 2.5 & 3.3 & $20^{*}$ \\
\hline 3 & 17 & 28.8 & 10.3 & 5.3 & 2.3 & & 15 \\
\hline 4 & 0 & 23.5 & 7.0 & 6.5 & 1.3 & & 16 \\
\hline \multicolumn{8}{|c|}{ Operated Control Group } \\
\hline 1 & 4 & 13.8 & 3.0 & & & & 8 \\
\hline 2 & 6 & 11.5 & 3.0 & & & & 8 \\
\hline 3 & 7 & 13.0 & 1.5 & & & & 7 \\
\hline \multicolumn{8}{|c|}{ Unoperated Control Group } \\
\hline 1 & 2 & 9.0 & 4.5 & & & & 8 \\
\hline 2 & 5 & 12.5 & 2.3 & 0.5 & & & 9 \\
\hline 3 & 5 & 8.3 & 1.5 & & & & 7 \\
\hline
\end{tabular}

*Failure to attain learning set criterion.

lary body lesions on any of the five reversal blocks. Thus, a one-tailed t-test comparison of the means of the experimental animals versus the two control groups (combined) on the first block of four reversals yielded a highly significant difference $[t(8)=7.86, p<.005]$. Similarly, a highly significant difference in performance was obtained between the experimental and control subjects on the second block of four reversals $[\mathrm{t}(8)=5.68, \mathrm{p}<.005]$.

There was no direct correlation between the amount of brain damage from surgery and subsequent performance on the spatial reversal task; that is, the subject with the least amount of resultant tissue damage also displayed the poorest learning performance.

In order to better understand the learning impairment on the spatial reversal task by the monkeys with mammillary-body lesions, we utilized a classification scheme, devised by Jones and Mishkin (1972), in which we subdivided the errors on the first eight reversals into three different categories. The first category, which would be indicative of a perseverative class of responses, consisted of blocks of 10 trials containing 7 or more errors. The second category, which would reflect a transitional period of learning between the gradual extinction of the previous response and the acquisition of a new response, consisted of 10 trial blocks containing from 4 to 6 errors (chance performance). The last category, which would be reflective of the acquisition of the new response just before the attainment of criterion performance, consisted of blocks of 10 trials containing only 2 or 3 errors. The frequency of occurrence of these three major types of errors is plotted for each group of subjects in Figure 3. It is evident from the figure that, although the monkeys with mammillary-body lesions did not differ from the two control groups (combined) in the number of errors during the mainten- ance of new responses before reaching criterion performance $(U=11, p>.10$, Mann-Whitney $U$ test), they displayed a significant increase in the number of perseverative errors and an even greater increase in the number of errors during the transition between the extinguishing of a former response and the maintenance of new responses $(U=0, p=.005$, MannWhitney U test).

The results of the object reversal task are shown in Figure 4. As indicated in the figure (and outlined in Table 2 for each animal), the monkeys with mammillary-body lesions were again found not to differ significantly from the operated $(U=5, p>.10$, Mann-Whitney $U$ test) or the unoperated $(U=5$, $p>.10$, Mann-Whitney $U$ test) control groups in the initial acquisition (trials to criterion) of the task, that is, object discrimination learning. As is also evident from the figure, the performance of subjects

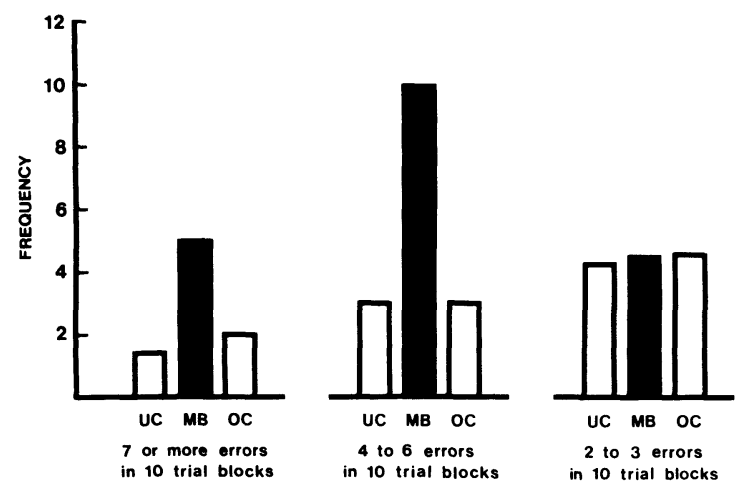

Figure 3. Mean frequency of occurrence for the three different classes of errors (as described in the text) for each subject group on the spatial reversal task. Abbreviations: $\mathrm{UC}=$ unoperated control group, $\mathrm{MB}=$ mammillary-body-lesion group, $\mathrm{OC}=\mathrm{op}$ erated control group. 


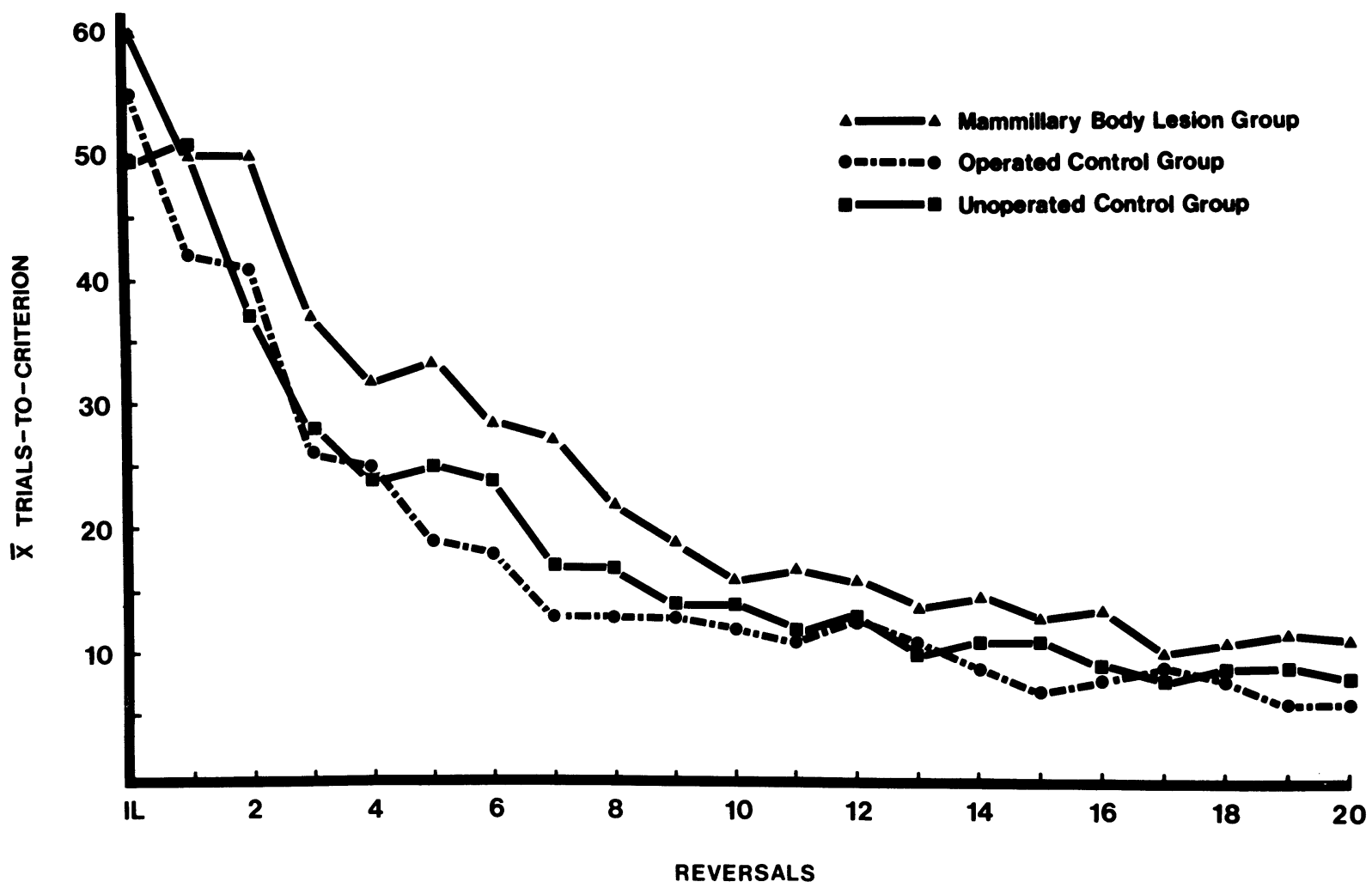

Figure 4. Object reversal learning curves. Abbreviations: $\mathrm{IL}=$ initial learning.

with mammillary-body lesions was not significantly different from that of the two control groups on the subsequent reversals. Using the same analysis of variance design used to assess spatial reversal performance, the overall difference between the subject groups was found not to be statistically significant $[F(2,7)=1.41, p>.10]$, although there was a significant effect across reversals $[F(19,133)=67.72$, $\mathrm{p}<.01]$. The interaction of the two main effects also proved to be insignificant $[F(38,133)=1.10, p>$ .10]. Surprisingly, the lack of a significant effect between groups was obtained even though the control subjects found this task to be more difficult than the spatial reversal task. That is, not only did the control subjects require more trials to reach criterion during the early reversals of this task, but also none of the subjects in the two control groups was able to attain the learning set criterion within 20 reversals.

The data for the individual animals on the object task is given in Table 2 in blocks of four reversals. As shown in the table, there was consistent overlap in performance between the three groups across the 20 reversals, with the subjects with mammillary-body lesions showing the greatest difficulty on the second block of problems.

Although the subject showing the poorest performance in the experimental groups also had the largest brain lesion, there was again no direct correlation between the amount of tissue damage and reversal performance.

\section{DISCUSSION}

In this study, we compared monkeys with mammillary-body lesions with matched operated and unoperated control monkeys on the acquisition of spatial and nonspatial reversal tasks in order to examine the effects of these lesions on the development of learning strategies as well as on the adjustment of cognitive strategies to changes in reinforcement contingencies. In the spatial reversal task, we found that although monkeys with mammillary-body lesions did not differ from the control animals in initially learning a positional response, they were markedly impaired in learning to periodically reverse acquired left-right spatial responses. In the nonspatial (object) task, however, the monkeys with mammillary-body lesions were found not to differ from the control groups either in initially acquiring a two-choice object discrimination or in subsequently learning to reverse responses between the two objects. Moreover, the object reversal task appeared to be much more difficult than the spatial reversal task, because none of the control subjects were able to attain the preset criterion level within the 20 reversals. Thus, it may not have been the ability to reverse responses per se 
Table 2

Object Reversal Learning

\begin{tabular}{|c|c|c|c|c|c|c|c|}
\hline \multirow[b]{2}{*}{ Subject } & \multirow{2}{*}{$\begin{array}{l}\text { Initial Learning } \\
\text { Trials to Criterion } \\
\end{array}$} & \multicolumn{5}{|c|}{$\begin{array}{c}\text { Blocks of Four Reversals } \\
\text { Trials to Criterion }\end{array}$} & \multirow{2}{*}{$\begin{array}{c}\text { Total Reversals to } \\
\text { Learning Set Criterion }\end{array}$} \\
\hline & & 1 & 2 & 3 & 4 & 5 & \\
\hline \multicolumn{8}{|c|}{ Mammillary Body Lèsion Group } \\
\hline $\begin{array}{l}1 \\
2 \\
3 \\
4\end{array}$ & $\begin{array}{r}112 \\
56 \\
22 \\
51\end{array}$ & $\begin{array}{l}58.5 \\
40.3 \\
37.8 \\
32.3\end{array}$ & $\begin{array}{l}38.0 \\
26.5 \\
28.3 \\
19.8\end{array}$ & $\begin{array}{r}28.0 \\
16.5 \\
9.3 \\
14.0\end{array}$ & $\begin{array}{r}20.8 \\
14.8 \\
10.5 \\
8.5\end{array}$ & $\begin{array}{r}16.8 \\
12.5 \\
7.8 \\
8.5\end{array}$ & $\begin{array}{l}20^{*} \\
20^{*} \\
20^{*} \\
20^{*}\end{array}$ \\
\hline \multicolumn{8}{|c|}{ Operated Control Group } \\
\hline $\begin{array}{l}1 \\
2 \\
3\end{array}$ & $\begin{array}{l}90 \\
41 \\
33\end{array}$ & $\begin{array}{l}38.0 \\
34.8 \\
27.0\end{array}$ & $\begin{array}{l}19.8 \\
12.8 \\
14.3\end{array}$ & $\begin{array}{r}17.5 \\
11.3 \\
8.0\end{array}$ & $\begin{array}{r}16.0 \\
6.3 \\
4.8\end{array}$ & $\begin{array}{r}13.8 \\
4.3 \\
4.8\end{array}$ & $\begin{array}{l}20 * \\
20 * \\
20 *\end{array}$ \\
\hline \multicolumn{8}{|c|}{ Unoperated Control Group } \\
\hline $\begin{array}{l}1 \\
2 \\
3\end{array}$ & $\begin{array}{l}74 \\
46 \\
27\end{array}$ & $\begin{array}{l}31.5 \\
41.0 \\
32.5\end{array}$ & $\begin{array}{l}21.3 \\
25.0 \\
16.0\end{array}$ & $\begin{array}{l}14.8 \\
14.5 \\
10.8\end{array}$ & $\begin{array}{r}13.8 \\
10.3 \\
7.5\end{array}$ & $\begin{array}{r}12.0 \\
8.5 \\
5.8\end{array}$ & $\begin{array}{l}20 * \\
20 * \\
20 *\end{array}$ \\
\hline
\end{tabular}

*Failure to attain learning set criterion.

that was the critical factor in the spatial task, but rather the spatial component of the problem. These results are consistent with our earlier findings on delayed alternation learning following mammillarybody lesions (Holmes et al., 1983) and supports the hypothesis that the role of the mammillary nuclei in cognition may be limited to the spatial domain.

There are several factors that can interfere with the acquisition of reversal learning sets, for example, response perseveration and a tendency to shift responses prematurely during acquisition. An analysis of the pattern of errors on the spatial reversal task showed that, although the monkeys with mammillarybody lesions did not differ from the two control groups in the number of errors committed during the maintenance of new responses before attaining criterion, they differed markedly from the controls in the number of perseverative errors and displayed an even greater percentage of errors during the transition between the extinguishing of old responses and the acquisition and maintenance of new responses (chance performance). One possible explanation for the initially large number of errors committed during chance performance is that the monkeys with mammillary-body lesions were abnormally sensitive to the effects of proactive interference during the spatial reversals and that this sensitivity was gradually reduced with subsequent practice. Interestingly, patients with alcoholic Korsakoff's syndrome, whose neuropathology usually involves the mammillary nuclei, have been shown to perform similarly on spatial reversal learning (Oscar-Berman \& ZolaMorgan, 1980) and have also been shown to be quite sensitive to the effects of proactive interference (see Butters, 1981, for a review).
Lesions of two other limbic structures, the postcommissural fornix and the hippocampus, which are anatomically associated with the mammillary nuclei, have also been reported (Jones \& Mishkin, 1972; Mahut, 1971, 1972) to have no effect on object reversal learning but a marked effect on spatial reversal learning by monkeys. In a further analysis of their data, however, Jones and Mishkin (1972) showed that the pattern of errors on the spatial reversal task following bilateral hippocampal removal (along with the fusiform-hippocampal gyrus) closely resembled that obtained from monkeys with orbitofrontal lesions in which the greatest percentage of errors during reversals was due to the perseveration of former responses. Thus, although both the hippocampus and the mammillary nuclei appear to be much more crucial for spatial than for nonspatial learning, their relative modulating influences on the acquisition of a spatial response may be quite different; that is, although the hippocampus may be more important for the initial shifting of responses, the mammillary bodies may be more important for the acquisition and maintenance of new responses. A possible differentiation of function in spatial cognition by structures contributing to the hippocampal-mammillary system would be an interesting problem to pursue in the future.

\section{REFERENCES}

Butters, N. The Wernicke-Korsakoff syndrome: A review of psychological, neuropathological, and etiological factors. In M. Galanter (Ed.), Currents in alcoholism (Vol. 8). New York: Grune \& Stratton, 1981.

Butters, N., \& Cermack, L. S. Alcoholic Korsakoff's syndrome: 
An information processing approach to amnesia. New York: Academic Press, 1980.

Gross, C. G. Inferotemporal cortex and vision. In E. Steller \& J. M. Sprague (Eds.), Progress in physiological psychology (Vol. 5). New York: Academic Press, 1973.

Holmes, E. J., Jacobson, S., Stein, B. M., \& Butters, N. Ablations of the mammillary nuclei in monkeys: Effects on post-operative memory. Experimental Neurology, 1983, 81, 97-113.

Jones, B., \& Mishkin, M. Limbic lesions and the problem of stimulus-reinforcement associations. Experimental Neurology, 1972, 36, 362-377.

KAPUR, N., \& BUtTers, N. Visuoperceptive deficits in long-term alcoholics and alcoholics with Korsakoff's psychosis. Journal of Studies on Alcohol, 1977, 38, 2025-2035.

MAHUT, H. Spatial and object reversal learning in monkeys with partial temporal lobe ablations. Neuropsychologia, 1971, 9, 409-424.

MahuT, $H$. A selective spatial deficit in monkeys after transection of the fornix. Neuropsychologia, 1972, 10, 65-74.

O'KeEfe, J., \& NADEL, L. The hippocampus as a cognitive map. Oxford: Clarendon Press, 1978.

Ocsar-Berman, M., Sahakian, B. J., \& Wikmark, G. Spa- tial probability learning by alcoholic Korsakoff patients. Journal of Experimental Psychology: Human Learning and Memory, 1976, 2, 215-222.

Oscar-Berman, M., \& Zola-Morgan, S. Comparative neuropsychology and Korsakoff's syndrome: I. Spatial and visual reversal learning. Neuropsychologia, 1980, 18, 499-512.

SNider, R. S., \& LEe, J. C. A stereotaxic atlas of the monkey brain (Macaca mulatta). Chicago: University of Chicago Press, 1961.

Thompson, R. A note on cortical and subcortical injuries and avoidance learning by rats. In J. M. Warren \& K. Akert (Eds.), The prefrontal granular cortex and behavior. New York: McGrawHill, 1964.

Thompson, R. Rapid forgetting of a spatial habit in rats with hippocampal lesions. Science, 1981, 212, 959-960.

Thompson, R., Langer, S. K., \& Rich, I. Lesions of the limbic system and short-term memory in albino rats. Brain, 1964, 87, 537-542.

Victor, M., Adams, R. D., \& Collins, G. H. The WernickeKorsakoff syndrome. Philadelphia: F. A. Davis, 1971.

(Manuscript received June 15, 1983;

revision accepted for publication August 23, 1983.) 